Questions vives

\section{Questions Vives}

Recherches en éducation

Vol. $9 n^{\circ} 19$ | 2013

Éducation routière, changement de comportement et formation à la conduite : constat, enjeux et transformations

\title{
Genre et rapport au risque : de la compréhension au levier pour l'action
}

Gender and risk assessment: from understanding to lever for action

\section{Marie-Axelle Granie}

\section{(2) OpenEdition}

Journals

Édition électronique

URL : http://journals.openedition.org/questionsvives/1273

DOI : 10.4000/questionsvives. 1273

ISBN : 978-2-8218-1394-6

ISSN : $1775-433 \mathrm{X}$

Éditeur

Université Aix-Marseille (AMU)

Édition imprimée

Date de publication : 15 juillet 2013

Pagination : 65-87

ISBN : 978-2-912643-43-8

ISSN : 1635-4079

Référence électronique

Marie-Axelle Granie, "Genre et rapport au risque : de la compréhension au levier pour l'action », Questions Vives [En ligne], Vol. 9 n¹9 | 2013, mis en ligne le 15 octobre 2013, consulté le 19 avril 2019. URL : http://journals.openedition.org/questionsvives/1273; DOI : 10.4000/questionsvives.1273

Ce document a été généré automatiquement le 19 avril 2019.

Questions Vives est mis à disposition selon les termes de la licence Creative Commons Attribution -

Pas d'Utilisation Commerciale - Pas de Modification 4.0 International. 


\title{
Genre et rapport au risque : de la compréhension au levier pour l'action
}

\author{
Gender and risk assessment: from understanding to lever for action
}

\author{
Marie-Axelle Granie
}

\section{Introduction}

1 De façon générale, les données accidentologiques pointent que les individus de sexe masculin, quel que soit leur âge, sont significativement plus impliqués dans une large variété d'activités risquées et physiquement dangereuses, activités qui sont un contributeur majeur de leur fréquence de traumatismes (Alkon et al., 2000 ; Bergdahl \& Norris, 2002 ; Byrnes et al., 1999; Hamming \& Weatherly, 2003 ; McQuillan \& Campbell, 2006 ; Romano et al., 2008 ; Rosen \& Peterson, 1990).

Dans les pays développés, les décès suivant des blessures non intentionnelles sont plus nombreux chez les garçons que chez les filles. Un rapport de l'UNICEF (2001) montre que les garçons de moins de 14 ans des pays de l'OCDE ont $70 \%$ de probabilité de plus que les filles de mourir dans un accident. Les garçons ont des accidents plus fréquents (Baker et al., 1992 ; Rivara \& Mueller, 1987) et plus graves (Rivara et al., 1982) que les filles, et l'exposition au risque ne semble pas être la seule variable explicative (Howarth et al., 1974 ; Waylen \& McKenna, 2002).

3 Cette différence entre sexes continue à se manifester à l'âge adulte, notamment dans les accidents routiers. En 2007, pour le même nombre de kilomètres parcourus, les femmes françaises avaient 3,7 fois moins de risque que les hommes d'être tuées, 1,7 fois moins de risque d'être blessées et 11,7 fois moins de risque d'être condamnées pour des délits routiers (ONISR, 2008). L'écart progresse jusqu'à atteindre un maximal de 8 hommes tués pour 2 femmes entre 35 et 39 ans ( 70 à $80 \%$ d'hommes parmi les tués sur la route entre 15 et 59 ans) (Assailly, 2001). Ce différentiel n'est pas propre à la France : dans la plupart des 
pays occidentaux les conducteurs ont 2 à 3 fois plus de risque de mourir lors d'un accident routier que les conductrices (Hanna et al., 2006 ; Nell, 2002; Özkan \& Lajunen, 2005). Au niveau mondial, près de trois fois plus d'hommes que de femmes meurent dans les accidents de la route, contribuant pour une très large part aux différences de sexe observées dans le nombre de traumatismes mortels non intentionnels (World Health Organization, 2002). Cette différence peut même dépasser un facteur 3 chez les 15-29 ans (Roads and Traffic Authority of New South Wales, 2001).

4 L'objectif de cet article est de faire un état des lieux des connaissances permettant de comprendre et de modéliser la relation entre sexe et accidentologie routière dans une approche psycho-socio-développementale, afin de proposer des pistes de changement pour le continuum éducatif en sécurité routière permettant de réduire le surrisque masculin.

\section{Différences de sexe dans le rapport au risque et à la règle}

5 Les différences d'exposition des hommes et des femmes ne suffisent pas à expliquer les écarts entre sexe dans l'accidentologie : les prises de risque et les infractions aux règles routières expliquent mieux les différences de sexe dans la mortalité routière que le nombre de kilomètres parcourus (Waylen \& McKenna, 2002). Une méta-analyse de 150 études portant sur les différences de sexe dans la prise de risque supporte clairement l'idée que les participants des recherches référencées ont plus tendance à prendre des risques que les participantes (Byrnes et al., 1999). Ces différences ne sont pas propres aux adultes et se manifestent dès l'enfance.

Les observations en situation naturelle ou en laboratoire montrent que les garçons s'engagent dans des comportements plus risqués que les filles (Coppens \& Gentry, 1991; Ginsburg \& Miller, 1982 ; Morrongiello \& Dawber, 1999 ; Rosen \& Peterson, 1990), même lorsqu'ils sont impliqués dans la même activité que celles-ci (Galligan \& Kuebli, 2011 ; Granié, 2007, 2011 ; Morrongiello \& Dawber, 1998; Rivara et al., 1982). Les jeunes conducteurs mâles rapportent plus de comportements risqués au volant (Harré et al., 1996) et plus d'attitudes risquées (Harré et al., 2000). Ainsi, près d'un-tiers des jeunes hommes prennent des risques pour le plaisir pendant la conduite, près de quatre fois plus que les femmes (Jessor, 1987). Le type d'accident lui-même varie en fonction du sexe, les hommes étant davantage impliqués dans des accidents consécutifs à des prises de risque, comme ceux comprenant un dépassement ou une perte de contrôle en courbe (Waylen \& McKenna, 2002).

7 Cette différence de sexes s'observe dans la prise de risque mais aussi dans l'évaluation du risque chez les enfants, les adolescents et les adultes (DeJoy, 1992; Harré et al., 2000; Peterson et al., 1997 ; Rosenbloom \& Wolf, 2002). Les filles ont un seuil de jugement du danger plus bas que les garçons (Hill et al., 2000). La perception des conséquences négatives prédit l'évaluation du risque chez les filles, tandis que celle-ci est prédite la sévérité des conséquences chez les garçons (Harris et al., 2006 ; Hillier \& Morrongiello, 1998). Dès l'âge de 6 ans, les enfants des deux sexes estiment que les garçons ont moins de risques d'accident que les filles, même s'ils sont engagés dans la même activité (Morrongiello et al., 2000). Les filles se sentent plus vulnérables et expriment davantage d'appréhension face au risque d'accident (Galligan \& Kuebli, 2011 ; Hillier \& Morrongiello, 
1998 ; Morrongiello, 1997 ; Morrongiello \& Rennie, 1998 ; Peterson et al., 1997). Les garçons estiment avoir moins de risque de blessures, perçoivent celles-ci comme moins graves (Morrongiello, 1997), attribuent leurs accidents au manque de chance et expriment plus d'optimisme comparatif que les filles (Morrongiello \& Rennie, 1998). Les hommes conducteurs, spécialement les plus jeunes, évaluent leurs compétences de conduite comme plus élevées (Farrow \& Brissing, 1990; Özkan \& Lajunen, 2006; Tronsmoen, 2008), ont un sentiment de sécurité plus grand lors de la conduite (Bergdahl, 2007) et utilisent davantage celle-ci pour augmenter leur sentiment d'auto-efficacité (Farrow \& Brissing, 1990).

8 Les différences de sexe dans l'accidentologie routière peuvent ainsi être comprises par la propension plus grande des mâles à s'engager dans des comportements à risque, mais elles sont également expliquées par la plus forte propension des hommes à transgresser les règles routières. Les hommes rapportent un nombre plus important d'infractions routières (Lonczak et al., 2007) et manifestent un niveau plus faible de motivations normatives à se conformer aux règles routières (Yagil, 1998). Les conducteurs déclarent davantage d'infractions, alors que les conductrices déclarent davantage d'erreurs (Aberg \& Rimmö, 1998 ; Blockey \& Hartley, 1995 ; Reason et al., 1990). Les comportements dangereux et l'implication accidentelle sont plus fréquemment liés à des transgressions de règles légales chez les hommes (Harré et al., 1996; Lawton et al., 1997 ; Simon \& Corbett, 1996). Ces différences ne concernent pas que les comportements des conducteurs, mais aussi ceux des piétons, chez l'adulte (Latrémouille et al., 2004 ; Moyano Diaz, 2002 ; Rosenbloom, 2009 ; Rosenbloom et al., 2004 ; Tom \& Granié, 2011 ; Yagil, 2000) comme chez l'enfant ou l'adolescent (Elliott \& Baughan, 2004 ; Granié, 2007, 2009).

De façon générale, cette différenciation sexuée dans la conformité se manifeste déjà dès la petite enfance, les filles étant plus conformes aux demandes et aux exigences des parents, des enseignants et des autres figures d'autorité (Feingold, 1994 ; Kochanska, 2002 ; Kochanska et al., 1995). Plus spécifiquement dans l'éducation au risque, la connaissance des prescriptions parentales ne découragent pas la prise de risque des garçons (Morrongiello \& Dawber, 2004) et les filles se conforment plus que les garçons aux demandes maternelles visant à éviter les objets dangereux (Morrongiello \& Dawber, 1998). Lors du déplacement piéton, les filles tiennent davantage la main de l'adulte (Granié, 2007 ; Zeedyk \& Kelly, 2003) mais sont également davantage initiatrices de la prise de main (Granié, 2007), les filles étant, de façon générale, plus soumises aux adultes (Block, 1983 ; Fagot, 1978 ; Ruble \& Martin, 1998 ; Turner \& Gervai, 1995).

10 L'ensemble de ces recherches montre des différences entre sexes en termes de blessures non-intentionnelles, de prises de risque et de conformité aux règles routières. Comprendre et expliquer l'ensemble de ces « sexospécificités » (Organisation Mondiale de la Santé, 2005), devient alors un enjeu majeur de santé publique, afin d'envisager de nouveaux leviers pour l'éducation routière en ciblant plus précisément les populations les plus à risque accidentel, pour leur proposer une formation plus adaptée.

11 Toutes ces recherches sont faites dans le cadre du paradigme des différences de sexe, cherchant à comprendre si, et à quel degré, les deux catégories de sexes - tels que définies par les caractéristiques biologiques, physiologiques et phénotypiques différentes des mâles et des femelles - diffèrent en termes de comportements (Eagly, 1995 ; Maccoby \& Jacklin, 1974 ; Merz, 1979). Même si leurs résultats sont informatifs dans le cadre de la compréhension de l'accidentologie dans l'espace routier, ils restent descriptifs d'un état 
de fait qu'il s'agit ensuite de dépasser afin de comprendre les origines de ces différences de sexe comme facteurs explicatifs de l'accidentologie.

\section{Du constat aux explications}

\subsection{Le biologique et le social}

12 La tendance des mâles à prendre plus de risques a d'abord été expliquée par une combinaison des théories biologiques et évolutionnistes (Lam \& Ozorio, 2013; Ronay \& von Hippel, 2010). La tendance à prendre des risques est notamment comprise comme une conséquence de la recherche de sensations (Rosenbloom \& Wolf, 2002 ; Zuckerman, 1991 ; Zuckerman et al., 1990), elle-même liée au niveau d'androgènes produit par le corps: les mâles prennent plus de risques parce qu'ils recherchent davantage les sensations, à cause d'une production d'androgènes plus importante (Social Issues Research Centre, 2004; Zuckerman, 1991). Ainsi, une augmentation du niveau de testostérone entraîne, chez les garçons mais aussi chez les filles, une augmentation de la sensibilité aux récompenses (les bénéfices) au détriment de la sensibilité aux punitions (les coûts) (van Honk et al., 2004), combinaison amenant une plus grande propension à la prise de risque (Coates \& Herbert, 2008; Dabbs \& Dabbs, 2000). La psychologie évolutionniste (Daly \& Wilson, 1987) pose par ailleurs que la prise de risque des mâles comme l'agression, la compétition ou l'infidélité - est une des conséquences naturelles des besoins de base du mâle de protéger la communauté et de s'assurer de la dissémination de ses gènes et du besoin des femelles de sélectionner le meilleur géniteur (Trivers, 1972).

Plus récemment, les recherches ont commencé à explorer les influences de l'environnement social sur les différences de sexes dans la prise de risque. Il s'agit en particulier de passer d'un questionnement en termes de différences de sexe, se concentrant sur les disparités comportementales entre les mâles et les femelles, à un questionnement en termes de genre, visant à comprendre les effets de la construction individuelle psychosociale de l'appartenance à un groupe de sexe.

Certains chercheurs ont ainsi investigué la conformité aux rôles sociaux de sexe, c'est-àdire aux attentes de comportement que développe le groupe social en fonction de l'appartenance de l'individu à un groupe de sexe (Basow, 1992), comme facteur explicatif des différences de sexe dans la prise de risque et la transgression (Byrnes et al., 1999; d'Acremont \& Van der Linden, 2006 ; Eagly, 1987 ; Rowe et al., 2004). La soumission est par exemple plus attendue du comportement féminin et l'indépendance plus attendue du comportement masculin (d'Acremont \& Van der Linden, 2006). Simon et Corbett (1996) posent ainsi que les différences de sexe dans la conduite sont l'expression de différences dans les rôles de sexe, le rôle féminin étant passif, non compétitif et prudent alors que le rôle masculin est risqueur, compétitif, agressif et non-conforme. Cette interprétation est cohérente avec les normes de sexe à l'égard de la prise de risque (Yagil, 1998): les recherches montrent que les adolescents considèrent que boire et conduire est plus acceptable pour les garçons que pour les filles (Rienzi et al., 1996) et que les jeunes conducteurs sont moins supervisés que les jeunes conductrices (Parker et al., 1992).

Les rôles de sexe sont basés sur des stéréotypes, c'est-à-dire des croyances sociales sur ce que signifie être un homme ou une femme et ce qui est valorisé pour chaque sexe en termes d'apparence physique, d'attitudes, d'intérêts, de traits psychologiques, de 
relations sociales et d'occupations (Ashmore et al., 1986 ; Deaux \& Lewis, 1984 ; Huston, 1983, 1985). Les stéréotypes de sexe sont fréquemment étudiés comme l'une des plus envahissantes et accessibles formes de pensée catégorielle (Brewer, 1988; Fiske \& Neuberg, 1990) et la première des trois catégories sociales (avec l'âge et la « race ») qui sont acquises chez l'enfant (Mackie et al., 1996). Les stéréotypes de sexe posent ainsi la prise de risque comme un comportement typiquement masculin (Bem, 1981; Kelling et al., 1976 ; Morrongiello \& Hogg, 2004).

À la croisée des explications biologiques et sociales, certains auteurs (Arnett, 1992 ; Arnett \& Jensen, 1994) suggèrent que le niveau de prise de risque d'un individu dépend de deux facteurs : d'un côté les tendances endogènes et de l'autre les restrictions dont font l'objet les prises de risque dans la culture de l'individu (lois, normes, pratiques éducatives, etc.). Les travaux les plus récents tentent-ils ainsi de montrer l'origine à la fois biologique et sociale - innée et acquise - des différences de sexe dans la prise de risque. Brown et al. (2013) pointent ainsi un double facteur de risque pour la population des hommes. Le sexe biologique mâle entraîne un niveau élevé d'androgènes, un moindre effet de l'alcool sur les performances psychomotrices et un développement neurocognitif plus lent amenant une moindre perception du risque et une augmentation de l'impulsivité et de la recherche de sensation à l'adolescence. Le genre psychosocial masculin amène une valorisation de la prise de risque, de l'agressivité et de la compétition, une plus forte consommation d'alcool et une plus grande exposition en termes de fréquence de conduite. De la même façon, la population des femmes bénéficient d'un double facteur protecteur face au risque accidentel : le sexe biologique femelle mais aussi le genre féminin constituent des freins aux facteurs biologiques et sociaux explicatifs de la prise de risque.

Sous la pression de l'environnement social et notamment des attentes issues de leurs différents milieux de vie depuis l'enfance (Bussey \& Bandura, 1999), les individus ont effectivement tendance à se construire en tant qu'individus sexués, en se positionnant par rapport aux stéréotypes de sexe et aux rôles de sexe. Pour autant, l'individu ne se conforme pas à tous les stéréotypes qui définissent socialement son groupe de sexe. Les hommes ne sont pas tous uniformément masculins et les femmes uniformément féminines. L'individu peut reconnaître son appartenance à l'un des deux groupes de sexe tout en se reconnaissant également dans des caractéristiques socialement attribuées à l'autre groupe de sexe (Bem, 1974 ; Bem, 1981). Ainsi, la relation de l'individu aux rôles et aux stéréotypes définissant son groupe de sexe - des hommes par rapport au masculin par exemple - mais aussi à ceux définissant l'autre groupe de sexe - des hommes par rapport au féminin par exemple - doit être investiguée, afin de mieux comprendre les différences inter- et intragroupes de sexe dans les comportements à risque.

\subsection{Masculinité et prise de risque dans l'espace routier}

18 La prise en compte des effets de la conformité aux stéréotypes de sexe pour expliquer les différences entre hommes et femmes dans le domaine de la santé et du risque est très récente. Les recherches ont par exemple étudié l'effet de la conformité aux stéréotypes masculins dominants - ceux définissant l'homme blanc, cadre supérieur, hétérosexuel et protestant aux Etats-Unis - sur les comportements de santé des hommes (Courtenay, 2000 ; Mahalik et al., 2007), ainsi que les effets de la masculinité sur la prise de risque dans le sport (Cazenave et al., 2003), dans la conduite automobile (Özkan \& Lajunen, 2006; Sibley \& Harré, 2009) et dans la prise de risque en général (Raithel, 2003). Leur effet a 
également été démontré sur le comportement effectif des hommes sur simulateur de conduite (Schmid Mast et al., 2008). Dans tous les cas étudiés, les recherches montrent que la conformité aux stéréotypes masculins - quel que soit le sexe biologique de l'individu - amène des comportements à risque accidentel plus fréquents que la conformité aux stéréotypes féminins.

Cet effet des stéréotypes de sexe sur la prise de risque s'observe dès l'enfance préscolaire. Le niveau de masculinité d'enfants de 3-6 ans des deux sexes a un effet sur leurs prises de risque et ces comportements à risque accidentel suivent le niveau de masculinité de l'enfant : stables avec l'âge chez les garçons, ils baissent chez les filles (Granié, 2010a). Cet effet de la masculinité sur les comportements à risque perdure à l'adolescence, la conformité aux stéréotypes masculins étant un renforçateur de la prise de risque déclarée en tant que piéton (Granié, 2009).

Confirmant en cela d'autres recherches (Raithel, 2003 ; Sarrasin \& Mayor, 2010), ces deux études confirment que la conformité aux stéréotypes masculins se révèle être un meilleur prédicteur de la prise de risque que le sexe biologique (Granié, 2009, 2010). Ainsi, ce n'est pas le fait d'être un garçon ou une fille qui prédit le niveau de prise de risque déclaré, mais le fait de se reconnaître - ou d'être reconnu - comme plus ou moins masculin, c'està-dire de manifester des comportements et des traits de personnalité que la société attribue au sexe masculin. En cela, les résultats de ces études confirment que la différence entre sexes dans la prise de risque peut être en partie attribuée à l'adhésion de l'individu aux stéréotypes définissant dans la société occidentale le comportement attendu du groupe masculin (Byrnes et al., 1999 ; d'Acremont \& Van der Linden, 2006 ; Rowe et al., 2004).

\subsection{De la corrélation à la causalité}

Toutefois, si ces recherches établissent un lien, elles ne permettent pas de valider une relation de causalité entre identité sexuée et prise de risque. Ainsi, reste-t-il à déterminer si c'est la conformité aux stéréotypes masculins qui amène principalement les hommes à prendre plus de risques que les femmes, ou si c'est le niveau de prise de risque qui est interprété, socialement et individuellement, comme un signe de masculinité.

Des recherches plus récentes répondent à cette question et portent sur l'influence de l'identité sexuée sur le comportement de l'adulte au volant de sa voiture, en activant les stéréotypes de sexe associés directement ou indirectement à la tâche de conduite, par attribution d'un contexte sexué à cette tâche (Schmid Mast et al., 2008) ou par activation d'un stéréotype de sexe par la consigne donnée préalablement à la tâche (Félonneau \& Becker, 2011; Yeung \& von Hippel, 2008). Dans l'étude de Schmid Mast et al. (2008), l'activation des stéréotypes de sexe - par l'énonciation au cours de la tâche de conduite de termes relevant du rôle de sexe masculin - engendre une augmentation des comportements à risque, comme une vitesse moyenne de conduite plus élevée. Dans l'étude de Yeung et Von Hippel (2008), le stéréotype négatif de la femme au volant est activé auprès de femmes conductrices lors de la consigne préalable à la tâche de conduite et a un effet perturbateur sur leurs performances au volant. Afin, dans l'étude de Félonneau et Becker (2011), l'évocation du stéréotype de la femme au volant abaisse les performances des femmes dans un test du code de la route. Ces trois recherches permettent d'établir que la prise de risque est sous l'influence de l'identité sexuée, plus 
précisément de la mobilisation de l'appartenance des individus à un groupe social de sexe et des comportements permettant à l'individu de manifester cette identité sociale.

L'ensemble de ces travaux montrent bien une relation entre le genre et les comportements à risque accidentel. Compte-tenu des types de risque mesurés, ils semblent également montrer une relation entre le genre et la conformité aux règles, puisque certaines de ces études se sont focalisées sur des risques relevant des comportements antisociaux et de l'infraction de règles légales.

\section{Genre et rapport à la règle : la prise en compte de l'internalisation}

Des travaux de ces cinq dernières années cherchent à montrer comment les différences de sexe dans les comportements routiers peuvent être expliquées par des différences dans la conformité engagée (Kochanska, 2002) et l'internalisation des règles (Granié, 2010b) - c'est-à-dire la transformation des règles sociales en comportements autorégulés (Grolnick et al., 1997) -. Deux études analysant les différences de sexe dans les comportements à risque dans l'espace routier montrent, pour l'une, que les filles préscolaires manifestent à la fois une plus grande connaissance des règles relatives aux comportement piéton, davantage de conformité à celles-ci et une plus grande internalisation que les garçons du même âge (Granié, 2007) et, pour l'autre, que les filles de 9-12 ans présentent davantage d'internalisation des règles relatives aux comportements à risque à vélo et ont tendance à présenter une moins grande propension à la prise de risque à vélo (Granié, 2011). Comme cela avait été montré chez les adultes piétons (Yagil, 1998, 2000), l'internalisation est plus importante chez les filles, ce qui suggère des plus hauts niveaux de motivation normatives (Tyler, 1990) chez ces dernières que chez les garçons. L'internalisation des règles semble ainsi être un facteur explicatif de la différence entre " mâles » et " femelles » dans les comportements à risque accidentel dans plusieurs domaines.

La construction de cette différence entre sexes dans l'internalisation des règles routières demande à être comprise. Comme pour la prise de risque, il s'agit alors d'expliquer les différences intergroupes et intragroupes de sexe dans la conformité en interrogeant la relation entre le niveau de conformité de l'individu aux stéréotypes de sexe et son niveau de conformité aux règles sociales.

Concernant les comportements d'infraction et de prise de risque en tant que piéton, les adultes ayant un fort niveau de masculinité ont, à propos des comportements transgressifs, des croyances normatives - c'est-à-dire la perception de la norme sociale (Huesmann \& Guerra, 1997) - plus faibles que les individus féminins (Granié, 2008). Ainsi, la masculinité entraîne une plus grande approbation de la prise de risque, qu'elle soit due à l'infraction ou au rapport de force avec l'automobiliste. La question est alors est de savoir si cette approbation des transgressions a un effet sur l'internalisation et la conformité aux règles routières.

Tostain, Georget et Lebreuilly (2005) ont montré que les enfants de 8-10 ans, garçons et filles, manifestant une forte conformité aux stéréotypes féminins déclarent plus de conformité aux règles piétonnes, ont des croyances normatives plus importantes et, pour justifier leur conformité, développent des arguments de type moral - par exemple « parce qu'il ne faut pas le faire». Les enfants masculins, pour justifier leur non-conformité, 
développent des arguments de type conventionnel, de nature consensuelle - par exemple en évoquant le fait que ces comportements transgressifs sont fréquents. Chez les adolescents, l'âge et la masculinité ont un effet direct de renforcement de la prise de risque, tandis que la féminité inhibe la prise de risque au travers de son effet sur la perception du danger et l'internalisation des règles (Granié, 2009).

Cette relation entre féminité et internalisation des règles peut s'expliquer par le fait que les traits de personnalité stéréotypiquement associés à la féminité (sensibilité aux besoins et aux sentiments d'autrui, chaleur, gentillesse, compréhension, tendresse et compassion (Bem, 1981)) sont autant de traits qui sont destinés à maintenir et apaiser le lien social, ramenant le rôle féminin à un rôle relationnel (Bakan, 1966 ; Parsons, 1955). En regard, les traits associés au masculin sont centrés soit sur le rapport de l'individu à lui-même (confiance en soi, sûr de soi, énergique, forte personnalité), soit l'amènent à entretenir une relation asymétrique avec autrui (agir en chef, diriger, prendre position, dominer, être en compétition) (Broverman et al., 1972). Ainsi, les individus féminins, en privilégiant le maintien de la relation à autrui, peuvent être amenés à se conformer aux règles sociales et à éviter les prises de risque davantage que les individus masculins, se reconnaissant plus dans des traits privilégiant l'individualité et la domination.

Il faut toutefois noter que toutes ces études sont basées sur des mesures autorapportées d'engagement dans des comportements à risque et que la construction psychologique de cette relation au cours du développement de l'individu n'a pas été encore abordée. Il peut également s'agir pour l'individu d'une recherche de cohérence entre une mesure autorapportée des comportements à risque et la perception des règles se rapportant aux mêmes comportements à risque accidentel (Yagil, 1998).

De plus, expliquer les différences de sexe dans la conduite par l'effet du genre part de l'hypothèse que l'activité de conduite serait une activité neutre que la conformité aux stéréotypes de sexe influencerait de l'extérieur, comme ils influencent d'autres comportements quotidiens (le comportement professionnel, par exemple). Pourtant, il peut être avancé qu'il existe des attentes sociales possiblement différenciées à l'égard de la conduite des hommes et des femmes, c'est-à-dire les stéréotypes associés aux hommes et aux femmes au volant.

\section{La conduite : une activité stéréotypée}

31 Si tout le monde connaît des proverbes populaires associés à la conduite des femmes, très peu de recherches ont pour l'instant permis de vérifier si ceux-ci incarnaient réellement des croyances sociales partagées en termes de comportements, susceptibles de guider les attentes sur le comportement d'autrui et d'orienter le comportement individuel.

Berger (1986) explique le développement d'un stéréotype sur la façon de conduire des femmes - contemporain de l'avènement de l'automobile individuelle - par la croyance sociale en l'incapacité des femmes à gérer les situations stressantes, demandant des prises de décisions rapides, comme le sont les situations routières. Pour autant, le contenu de ce stéréotype de la femme au volant n'a, à notre connaissance, été que peu exploré de façon approfondie (Félonneau \& Becker, 2011; Granié \& Pappafava, 2011), même si certaines recherches tendent à montrer qu'il est utilisé par les conducteurs (Davies \& Patel, 2005) et qu'il peut avoir des effets sur les comportements de conduite (Yeung \& von Hippel, 2008). 
33 Ainsi, les adolescents (Granié \& Pappafava, 2011) semblent considérer que les femmes sont naturellement inaptes à la conduite, leur prudence et leur conformité en étant une manifestation $\mathrm{Au}$ contraire, ils semblent estimer que le fait d'être un homme amène une compétence naturelle pour la conduite, leur infractionnisme en étant une manifestation. Ces principales caractéristiques vont dans le sens des stéréotypes posant les hommes comme plus agressifs et preneurs de risque (Locksley et al., 1980), plus compétents (DeJoy, 1992; Rothe, 1987 ; Stoddart, 1987) et les femmes comme moins actives et plus hésitantes (Harris \& Miller, 2000), ayant moins d'accidents, mais aussi moins de compétences de conduite (Glendon et al., 1996). Ils permettent également d'illustrer les résultats d'une étude de Näätänen et Summala (1976) qui a montré que la propension à conduire vite et à dépasser était généralement considérée comme une preuve de compétence.

Ainsi, l'inscription de la conduite dans le rôle de sexe masculin semble déterminer la capacité de conduite des individus de chaque sexe, dans une vision essentialiste des rôles de sexe (Heyman \& Giles, 2006; Prentice \& Miller, 2006 ; Tostain \& Lebreuilly, 2005). De plus, l'essence de la catégorie ne détermine pas seulement ce que ses membres sont, mais aussi ce qu'ils peuvent devenir, notamment au niveau de leurs compétences (Prentice \& Miller, 2006). Les hommes sont perçus comme des conducteurs naturellement compétents, comme les garçons sont perçus comme naturellement preneurs de risque (Morrongiello \& Dayler, 1996).

La conformité des garçons à l'image qu'ils ont construite de l'homme au volant peut expliquer pourquoi les garçons de 12-16 ans ont des attitudes plus risquées que les filles du même âge en termes de vitesses et de non port de la ceinture, avant même d'accéder à la conduite (Harré et al., 1996 ; Mann \& Lansdown, 2009), dans une culture dans laquelle la recherche du risque fait partie de la construction de la masculinité (Hopkins \& Emler, 1990).

Les relations entre identité sexuée, internalisation et comportements à risque ne sont pas linéaires. L'identité sexuée intervient comme prescriptrice de comportements plus ou moins risqués et, de ce fait, comme modulatrice de l'internalisation des règles. Ces travaux permettent de modéliser les mécanismes par lesquels s'expliquent la relation entre identité sexuée et prise de risque dans une perspective psycho-sociodéveloppementale.

\section{Les effets d'une double contrainte}

37 L'ensemble des études présentées montre que, en plus des différences biologiques entre sexes, les différences de prise de risque observables entre hommes et femmes dans l'espace routier sont dues à la manifestation chez les individus d'un comportement conformes aux attentes sociales (Ronay \& Kim, 2006), attentes également observables dans les stéréotypes de sexe associés à la conduite. Le niveau de conformité de l'individu aux stéréotypes masculins a un effet direct de renforcement de la prise de risque, celle-ci étant un comportement participant à la définition sociale de la masculinité. Le niveau de conformité de l'individu aux stéréotypes féminins joue également un rôle indirect : en entraînant un niveau d'internalisation plus élevé des règles routières, il inhibe la prise de risque. 

son comportement en tant que membre d'un groupe de sexe et celles régissant son comportement en tant qu'usager de l'espace routier. Parfois, ces normes sociales seront cohérentes, parfois elles seront en contradiction et l'individu aura alors à opérer des choix qui affecteront le poids de ces normes dans son système de valeurs personnel et participeront à la construction de sa personnalité (Tap, 1991). Ainsi les filles se trouvent en situation de cohérence entre les attentes sociales liées à leur appartenance sexuée et les attentes sociales liées à leur position d'usager. Dans les deux cas, sont valorisées la prudence et la conformité, les attentes sociales liées à la féminité renforçant les attentes sociales liées à l'espace routier, ce qui peut également expliquer cette forte internalisation des règles chez les individus féminins. Les garçons se retrouvent pris dans une forme de double contrainte en termes de conformité (Ayral, 2011) et peuvent ressentir une incohérence, qui semble réelle, entre les attentes sociales liées à leur sexe valorisant la prise de risque - et les attentes sociales liées à leur usage de l'espace routier, visant la conformité aux règles et la prudence. Cette inconsistance entre les demandes sociales peut également être un élément explicatif de leur manque d'internalisation des règles routières, qui résulterait d'une façon pour les garçons de mettre en cohérence ces différentes attentes sociales. Leur besoin de confirmer leur appartenance sexuée par un comportement risqué et transgressif les amène à remettre en cause la validité des règles routières. La sanction qui peut s'en suivre peut même être valorisante et recherchée, puisqu'elle permet de consolider leur identité masculine (Ayral, 2011).

Ainsi, l'apprentissage de l'espace routier, par la somme des interactions potentielles et des normes véhiculées, participe-t-il au processus de socialisation, puis de personnalisation de l'individu (Malrieu, 1995; Tap, 1992). Comment l'ensemble de ces résultats peuvent-ils être utilisés dans les politiques et les actions publiques en termes d'éducation routière et de prévention des accidents des usagers de la route?

\section{Leviers d'action pour l'éducation routière}

\subsection{Favoriser l'internalisation des règles routières}

Les résultats de l'ensemble de ces recherches montrent que les modes de communication utilisés dans l'éducation routière et les messages de prévention doivent être questionnés afin d'induire l'internalisation morale des règles routières.

Le rappel des règles, les références à une autorité extérieure et aux sanctions afférentes aux transgressions, comportements rassemblés dans les recherches sur les pratiques éducatives sous le terme d'affirmation de pouvoir (Py \& Somat, 1999), ne permettent pas de communiquer les justifications des obligations et des interdits et ne permettent pas de centrer l'individu sur les effets de son action sur son bien-être et celui d'autrui. L'affirmation de pouvoir - le rappel de la sanction, la peur du gendarme - permet d'obtenir à court terme une soumission complaisante et une compréhension des conventions sociales, parce qu'elle apporte à l'individu de l'information sur les régularités attendues dans l'environnement social, incluant la présence de règles et les attentes des autorités. Cependant, l'affirmation de pouvoir ne facilite pas le développement moral et l'internalisation (Kuczynski, 1984) et entraîne une soumission complaisante et contextuelle, c'est-à-dire une conformité contextuelle, due à la présence 
d'une figure d'autorité, sans engagement sincère, qui n'est pas associée à l'internalisation des normes (Kochanska, 2002).

Au contraire, les méthodes éducatives inductives, le raisonnement et les justifications, en particulier s'ils sont orientés vers les conséquences pour autrui - « tu risques de lui faire mal ", « tu peux causer un accident »- (Hoffman, 1970), sont associés à une plus grande internalisation, au développement de la prise en compte d'autrui (Zahn-Waxler \& Chapman, 1982) et à la perception des règles comme de niveau moral et permettent de développer la résistance à la tentation et la conformité engagée (Kuczynski, 1982). Ainsi, les pratiques éducatives amenant la perception des règles routières comme relevant du niveau moral permettent l'internalisation des règles dans le système de valeurs personnel et conduisent l'individu à une conformité engagée.

Suivant les résultats des différentes recherches exposées ici, renforcer l'utilisation des valeurs altruistes, socialement associées au rôle de sexe féminin, dans l'argumentaire de l'éducation routière, pourrait être un moyen de promouvoir l'internalisation des valeurs sociales permettant un comportement autocontrôlé et une conformité engagée face aux règles légales du code de la route.

\subsection{Atténuer les prises de risque des individus masculins}

44 Les résultats des recherches actuelles améliorent nos connaissances sur les mécanismes qui expliquent les différences de sexe dans la prise de risque. Ils confirment que cette différence peut être due à des tendances endogènes, innées, mais s'explique également par le développement de l'identité sexuée et la socialisation différenciée. Les comportements de prises de risque sont fortement reliés à la masculinité, pour les individus des deux sexes et non pas à des différences tempéramentales entre garçons et filles. De plus, la conformité et l'internalisation sont liées à la conformité aux stéréotypes féminins, mettant en avant la prise en compte d'autrui.

En termes pratiques, deux types de réponses peuvent être donnés face à ce constat: réduire la différenciation sexuée dans la prévention du risque chez les jeunes enfants et au contraire prendre en compte la différenciation déjà existante chez les adolescents.

\subsubsection{Diminuer les stéréotypes de sexe dans l'enfance}

En effet, les croyances et les attitudes stéréotypées en fonction du sexe sont le résultat de la fréquence de l'exposition de l'individu à des contextes sociaux qui offrent des contenus sexués, l'accumulation de ce type d'expérience amenant une activation automatique des catégories de sexe (Eckes \& Trautner, 2000). Katz et Walsh (1991) suggèrent en conséquence que c'est avec les enfants les plus jeunes que les tentatives de changement des attitudes liées aux rôles de sexe sont plus efficaces, avant que leurs connaissances sur les stéréotypes ne soient cristallisées.

47 Les études montrent par ailleurs que les stratégies éducatives ne sont pas toutes efficaces pour changer les attitudes stéréotypées. Ainsi, montrer les bénéfices des activités contrestéréotypées ou encourager ces activités n'a pas d'effet sur les enfants (Maccoby, 1998, 2000). Insister sur les capacités des femmes et combattre les idées sexistes peut même avoir un effet pervers à l'adolescence, puisque cela entraîne un renforcement des stéréotypes de sexe chez les garçons, en pleine construction identitaire (Guttentag \& Bray, 1976). Certaines stratégies semblent toutefois efficaces. Ainsi les interventions 
destinées à modifier les relations entre l'étiquette de sexe et les activités et comportements, c'est-à-dire les interventions amenant l'enfant à utiliser d'autres critères que le sexe pour classifier les comportements - par exemple ce n'est pas un conducteur viril, c'est un conducteur imprudent, ce n'est pas une femme au volant, c'est une conductrice -, semblent plus efficaces que les tentatives pour modifier directement le contenu des stéréotypes eux-mêmes, car elles permettent à l'enfant une flexibilité plus importante des connaissances sexuées (Bigler \& Liben, 1990, 1992).

Il semble par ailleurs très difficile d'obtenir un changement durable du comportement en agissant directement sur celui-ci (Bigler, 1999; Katz \& Boswell, 1986) mais les expérimentations qui ont été menées n'ont évidemment pas pu contrer les expériences stéréotypées de l'enfant en dehors du laboratoire. Ces expériences stéréotypées de l'enfant et l'insistance sociétale sur les différences de sexe amènent Bigler (1999) à conclure que les changements doivent concerner avant tout les messages sociétaux envoyés à l'enfant concernant la signification et l'importance du genre, avant que toute stratégie éducative et psychologique pour dépasser les stéréotypes de sexe soit réellement efficace.

Il semble ainsi que ce ne soit pas sur le comportement de l'enfant lui-même qu'il faille agir, mais, là encore, sur les modèles comportementaux auxquels il est quotidiennement confronté. En effet, les enfants dont les parents ont des attitudes non traditionnelles à l'égard des rôles de sexe et dont les pères sont fréquemment engagés dans des activités féminines (McHale et al., 1999; Tenenbaum \& Leaper, 2002; Turner \& Gervai, 1995), comme ceux dont les parents présentant une personnalité androgyne (Orlofsky, 1979), sont moins conscients des stéréotypes de sexe et moins stéréotypés dans leurs intérêts et leurs compétences.

50 Ainsi, pour les jeunes enfants, la prévention des accidents devrait être basée sur une éducation moins différenciée au risque, qui passe par une plus grande indifférenciation des comportements des adultes en tant que parents et que conducteurs. Virtuellement tous les agents de socialisation de l'enfant (parents, école, pairs) ont des attentes et des comportements différents envers l'enfant en fonction de son sexe (Bussey \& Bandura, 1999). Cette socialisation a un effet majeur sur le comportement de l'enfant. Ainsi, les agents de socialisation doivent-ils être sensibilisés aux aspects essentialistes de leurs croyances et à leur rôle prépondérant dans la construction des différences de sexe dans les comportements à risque accidentel. Le garçon, comme la fille, doit bénéficier d'une réelle éducation au risque et à la conformité. Éduquer le garçon au risque, c'est lui faire prendre conscience de sa propre vulnérabilité face aux dangers et de la nécessité d'un apprentissage des comportements à risque accidentel, conscience qu'ont déjà les filles, assez tôt (Hillier \& Morrongiello, 1998). Renforcer cette perception de vulnérabilité - qui est une réalité aussi bien chez les hommes que chez les femmes - permet indirectement d'agir sur les comportements, en modifiant les croyances associés à chaque sexe.

\subsubsection{Déconnecter masculinité et prise de risque}

51 Le genre est ainsi une construction sociale et culturelle et le contenu des stéréotypes de sexe est le reflet des observations des comportements des hommes et des femmes (Eagly, 1987; Eagly \& Wood, 2012 ; Wood \& Eagly, 2009, 2010). Ceci pourrait être utilisé pour changer les relations entre les rôles de sexe et les comportements à risque chez 
l'adolescent, au travers de l'éducation au risque et des campagnes de prévention dans les médias.

Dans ce sens, certaines des caractéristiques socialement liées au féminin, reliées à des comportements plus prudents, pourraient être également reliées aux caractéristiques masculines des modèles de rôles masculins. L'éducation au risque des garçons adolescents pourrait ainsi utiliser comme exemples les nombreux modèles mâles qui ne correspondent pas aux stéréotypes masculins concernant le risque, tout en étant socialement reconnus comme des hommes typiques.

Les modèles de rôles ont une double fonction d'information sur les stéréotypes de sexe (acquisition) et de reproduction comportementale (adoption) (Bussey \& Bandura, 1999). Dans l'éducation au risque, la mise en avant des caractéristiques plus féminines de modèles de rôles masculins pourrait permettre l'acquisition de stéréotypes de sexe modifiés et l'adoption de comportements moins risqués chez les adolescents. La littérature montre ainsi que l'observation du renforcement positif du comportement contre-stéréotypé d'un modèle de même sexe augmente l'imitation de ce modèle, surtout si le renforcement provient d'un adulte mâle (Katz \& Walsh, 1991). En effet, ces derniers manifestent à l'accoutumée plus de différenciation des sexes que les femmes (Tennenbaum \& Leaper, 2003) et sont en quelque sorte des gardiens des rôles de sexe (Katz \& Walsh, 1991), ce qui les positionne aussi comme les meilleurs agents de changements (Owen Blakemore et al., 2009). Une stratégie éducative possible consiste ainsi à fournir aux mâles adolescents des modèles de rôles masculins présentant des comportements contre-stéréotypés en termes de conduite - comportement prudent, respectueux d'autrui et des règles - tout en étant fortement renforcés positivement par d'autres hommes. Il ne s'agit pas d'attendre des hommes un comportement moins masculin et d'entraver la construction de leur identité sexuée, mais de changer leur perception de la masculinité : le courage, la prise de risque, peuvent se manifester sur d'autres terrains moins dangereux pour la sécurité de tous, ou consister, par exemple, à la résistance à la pression sociale face au risque, ou à la résistance plus générale à la tentation.

$\mathrm{Au}$ contraire, l'insistance des médias sur les prises de risques des garçons et des hommes peut malheureusement renforcer les croyances des adolescents et de leur parents et ainsi accroître les croyances essentialistes (Heyman \& Giles, 2006) et la socialisation différenciée au risque. Par exemple, la récente campagne de mars 2012 de la Direction de la Sécurité Routière, intitulée "le Manifeste des femmes ", veut inciter les femmes à jouer le rôle de pacificatrice de la conduite des hommes qui les entourent. Si l'idée est louable, les messages ne donnent toutefois aucune explication des différences de sexe dans l'accidentologie sur lesquelles ils s'appuient et peuvent ainsi tendre à donner la perception que ces différences sont innées, biologiques: les femmes, naturellement prudentes, doivent protéger les hommes, naturellement risqueurs, d'eux-mêmes.

Un point important d'achoppement doit toutefois être pris en compte. La littérature montre que les positions de pouvoir entraînent une plus forte adhésion aux stéréotypes masculins (Durand-Delvigne, 1995) et que la manifestation de comportements masculins peut être une façon pour les filles adolescentes de se valoriser socialement aux yeux des pairs des deux sexes (Rubi, 2005). La position défavorable des femmes dans certains groupes sociaux peut amener celles-ci à manifester préférentiellement des comportements relevant des stéréotypes masculins et développer alors davantage de comportements à risque. Cet élément mérite d'être pris en compte dans la prévention des 
conduites à risque dans l'environnement routier, qui devrait alors se centrer sur les individus masculins plutôt que sur les individus de sexe biologique mâle.

\section{BIBLIOGRAPHIE}

Aberg, L., \& Rimmö, P.-A. (1998). Dimensions of aberrant driver behaviour. Ergonomics, 41, 39-56.

Alkon, A., Ragland, D. R., Tschann, J. M., Genevro, J. L., Kaiser, P., \& Boyce, W. T. (2000). Injuries in child care centers: gender-environment interactions. Injury Prevention, 6, 214-218.

Arnett, J. (1992). Reckless behavior in adolescence : a developmental perspective. Developmental Review, 12, 339-373.

Arnett, J., \& Jensen, L. A. (1994). Socialization and risk behavior in two countries: Denmark and the United States. Youth and Society, 26, 3-22.

Ashmore, R. D., Del Boca, F. K., \& Wohlers, A. J. (1986). Gender stereotypes. In R. D. Ashmore \& F. K. Del Boca (Eds.), The social psychology of female-male relations: a critical analysis of central concepts (pp. 69-119). New York : Academic Press.

Assailly, J.-P. (2001). La mortalité chez les jeunes [Mortality among teenagers]. Paris : Que sais-je? P.U.F.

Ayral, S. (2011). La fabrique des garçons. Paris : PUF.

Bakan, D. (1966). The duality of human existence. Chicago : Rand Mc Nally.

Baker, S. P., O’Neill, B., \& Ginsburg, M. J. (1992). The injury fact book. New York: Oxford University Press.

Basow, S. A. (1992). Gender stereotypes and roles. Pacific Grove, CA: Brooks/Cole.

Bem, S. L. (1974). The measurement of psychological androgyny. Journal of consulting and clinical psychology, 42, 155-162.

Bem, S. L. (1981). Gender schema theory: a cognitive account of sex-typing. Psychological Review, 88 , 354-364.

Bergdahl, J. (2007). Ethnic and gender differences in attitudes toward driving. The Social Science Journal, 44, 91-97.

Bergdahl, J., \& Norris, M. R. (2002). Sex differences in single vehicle fatal crashes: a research note. The Social Science Journal, 39(2), 287-293.

Berger, M., L. (1986). Women drivers ! The emergence of folklore and stereotypic opinions concerning feminine automotive behavior. Women's Studies International Forum, 9(3), 257-263.

Bigler, R. S. (1999). Psychological interventions designed to counter sexism in children : empirical limitations and theoretical foundations. In W.B. Swann Jr \& J.H. Langlois (Eds.), Sexism and stereotypes in modern society : the gender science of Janet Taylor Spence (pp. 129-151). Washington, DC : American Psychological Association. 
Bigler, R. S., \& Liben, L. S. (1990). The role of attitudes and interventions in gender-schematic processing. Child Development, 61, 1440-1452.

Bigler, R. S., \& Liben, L. S. (1992). Cognitive mechanisms in children's gender stereotyping: theoretical and educational implications of cognitive-based interaction. Child Development, 63, 1351-1363.

Block, J. H. (1983). Differential premises arising from differential socialization of the sexes : some conjectures. Child Development, 54, 1335-1354.

Blockey, P. N., \& Hartley, L. R. (1995). Aberrant driving behavior: errors and violations. Ergonomics 38(9), 1759-1771.

Brewer, M. B. (1988). A dual-process model of impression formation. In T. K. Srull \& R. S. Wyer (Eds.), Advances in social cognition (Vol. 1, pp. 1-36). Hillsdale, NJ: Lawrence Erlbaum.

Broverman, I., Vogel, S., Broverman, D., Clarkson, F., \& Rosenkrantz, P. (1972). Sex-role stereotypes : a current appraisal. Journal of Social Issues, 28, 59-78.

Brown, T. G. (2013). Sex Differences in First-Time DWI Offenders: Role of Alcohol and Neurobiological Factors. Paper presented at the Transportation Research Board 92nd annual meeting.

Bussey, K., \& Bandura, A. (1999). Social Cognitive Theory of Gender Development and Differentiation. Psychological Review, 106(4), 676-713.

Byrnes, J. P., Miller, D. C., \& Schafer, W. D. (1999). Gender differences in risk taking : a metaanalysis. Psychological Bulletin, 125(3), 367-383.

Cazenave, N., Le Scanff, C., \& Woodman, T. (2003). Spécificité de la prise de risque chez les femmes. $10^{\mathrm{e}}$ Congrès de l'Association des Chercheurs en Activités Physiques et Sportives (ACAPS), Toulouse.

Coates, J. M., \& Herbert, J. (2008). Endogenous steroids and financial risk taking on a London trading floor. Proceedings of the National Academy of Sciences, USA, 105, 6167-6172.

Coppens, N., \& Gentry, L. (1991). Video analysis of play-ground injury-risk situations. Research in Nursing and Health, 14, 129-136.

Courtenay, W. H. (2000). Constructions of masculinity and their influence on men's well-being: a theory of gender and health. Social Science \& Medicine, 50, 1385-1401.

d'Acremont, M., \& Van der Linden, M. (2006). Gender differences in two decision-making tasks in a community sample of adolescents. International Journal of Behavioral Development, 30(4), 352-358.

Dabbs, J. M., \& Dabbs, M. G. (2000). Heroes, rogues and lovers: Testosterone and behavior. New York: McGraw-Hill.

Daly, M., \& Wilson, M. (1987). Evolutionary psychology and family violence. In C. Crawford, M. Smith \& D. Krebs (Eds.), Sociobiology and Psychology: ideas issues and applications (pp. 293-309). Hillsdale, NJ: Erlbaum.

Davies, G. M., \& Patel, D. (2005). The influence of car and driver stereotypes on attributions of vehicle speed, position on the road and culpability in a road accident scenario. Legal and Criminological Psychology, 10, 45-62.

Deaux, K., \& Lewis, L. L. (1984). Structure of gender stereotypes: interrelations among components and gender labels. Journal of Personality and Social Psychology, 46(5), 991-1004.

DeJoy, D. M. (1992). An examination of gender differences in traffic accident risk perception. Accident Analysis \& Prevention, 24, 237-246. 
Durand-Delvigne, A. (1995). Pouvoir et genre. In EPHESIA (Ed.), La place des femmes. Les enjeux de l'identité et de l'égalité au regard des sciences sociales (pp. 453-456). Paris : La Découverte.

Eagly, A. H. (1987). Sex differences in social behavior : A social-role interpretation. Hillsdale, NJ : Erlbaum.

Eagly, A. H. (1995). The science and politics of comparing women and men. American Psychologist, 50(3), 145-158.

Eagly, A. H., \& Wood, W. (2012). Social role theory. In P. A. Van Lange, A. W. Kruglanski \& E. T. Higgins (Eds.), Handbook of theories of social psychology (Vol. 2, pp. 458-477). London: Sage Publications.

Eckes, T., \& Trautner, H. M. (2000). The developmental social psychology of gender : an integrative framework. In T. Eckes \& H. M. Trautner (Eds.), The developmental social psychology of gender (pp. 3-32). Mahwah, NJ : Lawrence Erlbaum.

Elliott, M. A., \& Baughan, C. J. (2004). Developing a self-report method for investigating adolescent road user behavior. Transportation Research Part F: Traffic Psychology and Behaviour, 7(6), 373-393.

Fagot, B. I. (1978). The influence of sex of child on parental reactions to toddler children. Child Development, 49, 459-465.

Farrow, J. A., \& Brissing, P. (1990). Risk for DWI: A New Look at Gender Differences in Drinking and Driving Influences, Experiences, and Attitudes among New Adolescent Drivers. Health Education \& Behavior, 17(2), 213-221.

Feingold, A. (1994). Gender differences in personality: a meta-analysis. Psychological Bulletin, 116 (3), 429-456.

Félonneau, M.-1., \& Becker, M. (2011). "Femmes au volant, danger au tournant". Les conductrices sont-elles victimes d'une menace du stéréotype ? Psychologie du Travail et des Organisations, 14(4), 314-329.

Fiske, S. T., \& Neuberg, S. L. (1990). A continuum model of impression formation from categorybased to individuating response: Influences of information and motivation on attention and interpretation. In M. P. Zanna (Ed.), Advances in experimental social psychology (Vol. 23, pp. 1-108). New-York, NY: Academic Press.

Galligan, K. M., \& Kuebli, J. E. (2011). Preschoolers' perceptions of their mothers' and fathers' reactions to injury-risk behavior. Accident Analysis \& Prevention, 43, 1316-1322.

Ginsburg, H. J., \& Miller, S. M. (1982). Sex differences in children's risk-taking behavior. Child Development, 53, 426-428.

Glendon, A. I., Dorn, L., Davis, R., Matthews, G., \& Taylor, R. G. (1996). Age and gender differences in perceived accident likelihood and driver competences. Risk Analysis, 16(755-762).

Granié, M.-A. (2008). Influence de l'adhésion aux stéréotypes de sexe sur la perception des comportements piétons chez l'adulte. Recherche - Transports - Sécurité, 101, 253-264.

Granié, M. A. (2007). Gender differences in preschool children's declared and behavioral compliance with pedestrian rules. Transportation Research Part F: Traffic Psychology and Behaviour, 10 (5), 371-382.

Granié, M. A. (2009). Sex differences, effects of sex-stereotype conformity, age and internalization on risk-taking among pedestrian adolescents. Safety Science, 47, 1277-1283. 
Granié, M. A. (2010a). Gender stereotype conformity and age as determinants of preschoolers' injury-risk behaviors. Accident Analysis \& Prevention, 42(2), 726-733.

Granié, M. A. (2010b). Socialisation au risque et construction sociale des comportements de l'enfant piéton : éléments de réflexion pour l'éducation routière. Enfances, Familles, Générations, 12, 88-110.

Granié, M. A. (2011). Différences de sexe et rôle de l'internalisation des règles sur la propension des enfants à prendre des risques à vélo. Recherche - Transports - Sécurité, 27, 34-41.

Granié, M. A., \& Pappafava, E. (2011). Gender stereotypes associated with vehicle driving among French preadolescents and adolescents. Transportation Research Part F : Traffic Psychology and Behaviour, 14(5), 341-353.

Grolnick, W. S., Deci, E. L., \& Ryan, R. M. (1997). Internalization within the family : the selfdetermination theory perspective. In J. E. Grusec \& L. Kuczynski (Eds.), Parenting and children's internalization of values (pp. 135-161). New York: John Wiley.

Guttentag, M., \& Bray, H. (1976). Undoing sex stereotypes. Research and ressources for educators. NewYork: McGraw-Hill.

Hamming, B., \& Weatherly, J. (2003). Gender and geographic differences in intentional and unintentional injury mortality among children in Illinois, 1988-1998. Injury Control and Safety Promotion, 10(4), 247-250.

Hanna, C. L., Taylor, D. M., Sheppard, M. A., \& Laflamme, L. (2006). Fatal crashes involving young unlicensed drivers in the US. Journal of Safety Research, 37, 385-393.

Harré, N., Brandt, T., \& Dawe, M. (2000). The Development of Risky Driving in Adolescence. Journal of Safety Research, 31(4), 185-194.

Harré, N., Field, J., \& Kirkwood, B. (1996). Gender differences and areas of common concern in the driving behaviors and attitudes of adolescents. Journal of Safety Research, 27(3), 163-173.

Harris, C. R., Jenkins, M., \& Glaser, D. (2006). Gender differences in risk assessment: why do women take fewer risks than men? Judgment \& Decision Making, 1, 48-63.

Harris, M. B., \& Miller, K. C. (2000). Gender and perception of gender. Sex Roles, 43, 843-863. Heyman, G. D., \& Giles, J. W. (2006). Gender and Psychological Essentialism. Enfance, 3, 293-310. Hill, R., Lewis, V., \& Dunbar, G. (2000). Young children's concepts of danger. British Journal of Developmental Psychology, 18(1), 103-119.

Hillier, L. M., \& Morrongiello, B. A. (1998). Age and gender differences in school-age children's appraisals of injury risk. Journal of Pediatric Psychology, 23(4), 229-238.

Hoffman, M. L. (1970). Moral Development. In P. Mussen (Ed.), Carmichael's manuel of child psychology (Vol. 2, pp. 261-300). New-York: Wiley.

Hopkins, N., \& Emler, N. (1990). Social network participation and problem behavior in adolescence. In K. Hurrelman \& F. Lösel (Eds.), Health hazards in adolescence (pp. 385-407). Berlin: de Gruyter.

Howarth, C. I., Routledge, D. A., \& Repetto-Wright, R. (1974). An analysis of road accidents involving child pedestrians. Ergonomics, 17(3), 319-330.

Huesmann, L. R., \& Guerra, N. G. (1997). Children's normative beliefs about aggression and aggressive behavior. Journal of Personality and Social Psychology, 72(2), 408-419. 
Huston, A. (1983). Sex-typing. In E. M. Hetherington (Ed.), Handbook of Child Psychology. vol. IV Socialization Personality and social development (Vol. vol. IV Socialization Personality and social development, pp. 387-468). New-York: Wiley.

Huston, A. (1985). The development of sex-typing: themes from recent research. Developmental Review, 5, 1-17.

Jessor, R. (1987). Risky driving and adolescent problem behavior: An extension of problem behavior theory. Alcohol, Drugs, and Driving, 3(1-13).

Katz, P. A., \& Boswell, S. (1986). Flexibility and traditionality in children's gender roles. Genetic social and general psychology Monographs, 112, 105-147.

Katz, P. A., \& Walsh, P. V. (1991). Modification of children's gender-stereotyped behavior. Child Development, 62, 338-351.

Kelling, G. W., Zirkes, R., \& Myerowitz, D. (1976). Risk as value: a switch of set hypothesis. Psychological Reports, 38, 655-658.

Kochanska, G. (2002). Committed compliance, moral self and internalization: a mediational model. Developmental Psychology, 38, 339-351.

Kochanska, G., Casey, R. J., \& Fukumoto, A. (1995). Toddlers' sensitivity to standard violations. Child Development, 66, 643-656.

Kuczynski, L. (1982). Intensity and orientation reasoning: motivational determinants of children's compliance to verbal rationales. Journal of Experimental Child Psychology, 34, 357-370.

Kuczynski, L. (1984). Socialization goals and mother-child interaction: strategies for long-term and short term compliance. Developmental Psychology, 20, 1061-1073.

Lam, D., \& Ozorio, B. (2013). The effect of prior outcomes on gender risk-taking differences. Journal of Risk Research.

Latrémouille, M.-E., Thouez, J.-P., Ranou, A., Bergeron, J., Bourbeau, R., \& Bussière, Y. (2004). Le sexe est-il une variable pertinente pour l'étude du comportement des piétons en intersection urbaine ? Recherche - Transports - Sécurité, 84, 171-188.

Lawton, R., Parker, D., Stradling, S. G., \& Manstead, A. S. R. (1997). Predicting road traffic accidents : The role of social deviance and violations. British Journal of Psychology, 88(2), 249-262.

Locksley, A., Borbiga, E., Brekke, N., \& Hepburn, C. (1980). Sex stereotypes and social judgement. Journal of Personality and Social Psychology, 39, 821-831.

Lonczak, H. S., Neighbors, C., \& Donovan, D. M. (2007). Predicting risky and angry driving as a function of gender. Accident Analysis \& Prevention, 39, 536-545.

Maccoby, E. E. (1998). The two sexes: growing up apart, coming together. Cambridge: Harvard University Press.

Maccoby, E. E. (2000). Perspectives on gender development. International Journal of Behavioral Development, 24(4), 398-406.

Maccoby, E. E., \& Jacklin, C. N. (1974). On the origins of psychological sex differences. In E. E. Maccoby \& C. N. Jacklin (Eds.), The psychology of sex differences (pp. 275-376). Stanford : Stanford University Press.

Mackie, D. M., Hamilton, D. L., Susskind, J., \& Rosselli, F. (1996). Social psychological foundations of stereotype formation. In C. N. Macrae, C. Stangor \& M. Hewstone (Eds.), Stereotypes and stereotyping (pp. 41-78). New-York: Guilford. 
Mahalik, J., Burns, S., \& Syzdek, M. (2007). Masculinity and perceived normative health behaviors as predictors of men's health behaviors. Social Science \& Medicine, 64(11), 2201-2209.

Malrieu, P. (1995). La personnalisation : analyse psycho-sociale. Apprendizagem / Desenvolvimento, IV(15/16), 9-17.

Mann, H. N., \& Lansdown, T. (2009). Pre-driving adolescent attitudes: Can they change? Transportation Research Part F : Traffic Psychology and Behaviour, 12, 395-403.

McHale, S. M., Crouter, A. C., \& Tucker, C. J. (1999). Family context and gender role socialization in middle childhood: Comparing girls to boys and sisters to brothers. Child Development, 70 , 990-1104.

McQuillan, R., \& Campbell, H. (2006). Gender differences in adolescent injury characteristics : A population-based study of hospital A\&E data. Public Health, 120, 732-741.

Merz, F. (1979). Geschelchtsunterschiede und ihre Entwicklung [Sex differences and their development]. Göttingen, Germany: Hogrefe.

Morrongiello, B. A. (1997). Children's perspectives on injury and close-call experiences : sex differences in injury-outcome processes. Journal of Pediatric Psychology, 22(4), 499-512.

Morrongiello, B. A., \& Dawber, T. (1998). Toddlers' and mothers' behaviors in an injury-risk situation : implications for sex differences in childhood injuries. Journal of Applied Developmental Psychology, 19(4), 625-639.

Morrongiello, B. A., \& Dawber, T. (1999). Parental influences on toddlers' injury-risk behaviors : are sons and daughters socialized differently? Journal of Applied Developmental Psychology, 20(2), 227-251.

Morrongiello, B. A., \& Dawber, T. (2004). Identifying factors that relate to children's risk taking decisions. Canadian Journal of Behavioural Science, 36(4), 255-266.

Morrongiello, B. A., \& Dayler, L. (1996). A community-based study of parents' knowledge, attitudes and beliefs related to childhood injuries. Canadian Journal of Public Health, 87(6), 383-388.

Morrongiello, B. A., \& Hogg, K. (2004). Mother's reactions to children misbehaving in ways that can lead to injury: implications for gender differences in children risk taking and injuries. Sex Roles, 50(1-2), 103-118.

Morrongiello, B. A., Midgett, C., \& Stanton, K.-L. (2000). Gender Biases in Children's Appraisals of Injury Risk and Other Children's Risk-Taking Behaviors. Journal of Experimental Child Psychology, 77 (4), 317-336.

Morrongiello, B. A., \& Rennie, H. (1998). Why do boys engage in more risk taking than girls ? The role of attributions, beliefs, and risk appraisals. Journal of Pediatric Psychology, 23(1), 33-43.

Moyano Diaz, E. (2002). Theory of planned behavior and pedestrians' intentions to violate traffic regulations. Transportation Research Part F : Traffic Psychology and Behaviour, 5(3), 169-175.

Näätänen, R., \& Summala, H. (1976). Road user behavior and trafic accidents. Amsterdam : North Holland.

Nell, V. (2002). Why young men drive dangerously: implications for injury prevention. Current Directions in Psychological Science, 11, 75-79.

ONISR. (2008). La Sécurité Routière en France. Bilan de l'année 2007. Paris : La Documentation Française. 
Organisation Mondiale de la Santé. (2005). Sexospécificité, femmes et santé: prendre en compte la dimension sexospécifique dans les politiques et les programmes de l'OMS. Genève : OMS.

Orlofsky, J. L. (1979). Parental Antecedents of Sex-Role Orientation in College Men and Women. Sex Roles, 5(4), 495-512.

Owen Blakemore, J. E., Berenbaum, S. A., \& Liben, L. S. (2009). Gender development. New-York: Psychology Press.

Özkan, T., \& Lajunen, T. (2005). Why are there sex differences in risky driving? The relationship between sex and gender-role on aggressive driving, traffic offences, and accident involvement among young turkish drivers. Aggresive Behavior, 31(6), 547-558.

Özkan, T., \& Lajunen, T. (2006). What causes the differences in driving between young men and women? The effects of gender roles and sex on young drivers' driving behaviour and selfassessment of skills. Transportation Research Part F: Traffic Psychology and Behaviour, 9, 269-277.

Parker, D., Manstead, A. S. R., Stradling, S. G., \& Reason, J. T. (1992). Determinants of intention to commit driving violations. Accident Analysis \& Prevention, 24(2), 117-131.

Parsons, T. (1955). Family socialization and interaction process. New-York : The Free Press of Glencoe. Peterson, L., Brazeal, T., Oliver, K., \& Bull, C. (1997). Gender and developmental patterns of affect, belief, and behavior in simulated injury events. Journal of Applied Developmental Psychology, 18(4), 531-546.

Prentice, D. A., \& Miller, D. T. (2006). Essentializing Differences Between Women and Men. Psychological Science, 17, 129-135.

Py, J., \& Somat, A. (1999). Discipline et obéissance en famille. In J. L. Beauvois, D. Dubois \& W. Doise (Eds.), La construction sociale de la personne (pp. 228-231). Grenoble : PUG.

Raithel, J. (2003). Risikobezogenes Verhalten und Geschlechtsrollenorientierung im Jugendalter [Risk-taking behavior and gender role orientation in adolescents]. Zeitschrift für Gesundheitspsychologie, 11(1), 21-28.

Reason, J. T., Manstead, A. S. R., Stradling, S., Baxter, J. S., \& Campbell, K. (1990). Errors and violations on the roads: a real distinction? Ergonomics, 33(10/11), 1315-1332.

Rienzi, B. M., McMillin, J. D., Dickson, C. L., \& Crauthers, D. (1996). Gender differences regarding peer influence and attitude toward substance abuse. Journal of Drug Education, 26, 339-347.

Rivara, F. P., Bergman, A. B., LoGerfo, J., \& Weiss, M. (1982). Epidemiology of childhood injury II : sex differences in injury rates. Developmental and Behavioral Pediatrics, 16, 362-370.

Rivara, F. P., \& Mueller, B. A. (1987). The epidemiology and causes of childhood injury. Journal of Social Issues, 43, 13-32.

Roads and Traffic Authority of New South Wales. (2001). Road traffic accidents in New South Wales. Haymarket, Australia: Roads and Traffic Authority, Road Safety Strategy Branch.

Romano, E., Kelley-Baker, T., \& Voas, R. B. (2008). Female involvement in fatal crashes : Increasingly riskier or increasingly exposed ? Accident Analysis \& Prevention, 40, 1781-1788.

Ronay, R., \& Kim, D.-Y. (2006). Gender Differences in Explicit and Implicit Risk Attitudes : A socially Facilitated Phenomenon. British Journal of Social Psychology, 45(2), 397-419.

Ronay, R., \& von Hippel, W. (2010). The Presence of an Attractive Woman Elevates Testosterone and Physical Risk Taking in Young Men. Social Psychological and Personality Science, 1(1), 57-64. 
Rosen, B. N., \& Peterson, L. (1990). Gender differences in children's outdoor play injuries : A review and an integration. Clinical Psychology Review, 10(2), 187-205.

Rosenbloom, T. (2009). Crossing at a red light: Behaviour of individuals and groups. Transportation Research Part F: Traffic Psychology and Behaviour, 12, 389-394.

Rosenbloom, T., Nemrodov, D., \& Barkan, H. (2004). For heaven's sake follow the rules : pedestrians' behavior in an ultra-orthodox and a non-orthodox city. Transportation Research Part F: Traffic Psychology and Behaviour, 7(6), 395-404.

Rosenbloom, T., \& Wolf, Y. (2002). Sensation seeking and detection of risky road signals : a developmental perspective. Accident Analysis \& Prevention, 34(5), 569-580.

Rothe, J. P. (1987). Erlebnis of young drivers involved in injury producing crashes. In J. P. Rothe (Ed.), Rethinking young drivers (pp. 199-252). New Brunswick : Transaction.

Rowe, R., Maughan, B., \& Goodman, R. M. (2004). Childhood psychiatric disorder and unintentional injury : findings from a national cohort study. Journal of Pediatric Psychology, 29(2), 119-130.

Rubi, S. (2005). Les «crapuleuses », ces adolescentes déviantes. Paris : PUF.

Ruble, D. N., \& Martin, C. L. (1998). Gender Development. In W. Damon \& N. Eisenberg (Eds.), Handbook of Child Psychology. vol. 3: Social, Emotional, and Personality Development (5th edition ed., pp. 933-1016). New-York: John Wiley \& Sons.

Sarrasin, O., \& Mayor, E. (2010, May). Cognitive appraisal and risk perception: what does really matter, biological sex or typical gender traits? Paper presented at the Gender Studies - Work in Progress Meeting. Interdisziplinäres Zentrum für Geschlechterforschung, University of Bern, Switzerland.

Schmid Mast, M., Sieverding, M., Esslen, M., Graber, K., \& Jäncke, L. (2008). Masculinity causes speeding in young men. Accident Analysis \& Prevention, 40, 840-842.

Sibley, C. G., \& Harré, N. (2009). A gender role socialization model of explicit and implicit biases in driving self-enhancement. Transportation Research Part F: Traffic Psychology and Behaviour, 12, 452-461.

Simon, F., \& Corbett, C. (1996). Road traffic offending, stress, age, and accident history among male and female drivers. Ergonomics, 39, 757-780.

Social Issues Research Centre. (2004). Sex differences in driving and insurance risk. An analysis of the social and psychological differences between men and women that are relevant to their driving behaviour. Oxford: Social Issues Research Centre.

Stoddart, K. (1987). Erfahrung of young drivers. In J. P. Rothe (Ed.), Rethinking young drivers (pp. 131-198). New Brunswick : Transaction.

Tap, P. (1991). Socialisation et construction de l'identité personnelle. In H. Malewska-Peyre \& P. Tap (Eds.), La socialisation de l'enfance à l'adolescence (pp. 49-73). Paris : P.U.F.

Tap, P. (1992). Personnalisation et conflits d'identité à l'adolescence. Apprendizagem / Desenvolvimento, III(10), 77-84.

Tenenbaum, H. R., \& Leaper, C. (2002). Are parents' gender schemas related to their children's gender-related cognitions? A meta-analysis. Developmental Psychology, 38(4), 615-630.

Tennenbaum, H. R., \& Leaper, C. (2003). Parent-child conversations about science: The socialization of gender inequities? Developmental Psychology, 39(1), 34-47. 
Tom, A., \& Granié, M. A. (2011). Gender Differences in Pedestrian Rule Compliance and Visual Search at Signalized and Unsignalized Crossroads. Accident Analysis \& Prevention, 43(5), 1794-1801. Tostain, M., \& Lebreuilly, J. (2005). Essentialisme enfantin lié au genre et prise en compte de l'environnement social : Une étude auprès d'enfants de cinq à onze ans. Archives de psychologie, 71 (278-279), 217-227.

Trivers, R. L. (1972). Parental investment and sexual selection. In B. Campbell (Ed.), Sexual selection and the descent of man. Chicago: Aldine.

Tronsmoen, T. (2008). Associations between self-assessment of driving ability, driver training and crash involvement among young drivers. Transportation Research Part F: Traffic Psychology and Behaviour, 11, 334-346.

Turner, P. J., \& Gervai, J. (1995). A multidimensional study of gender typing in preschool children and their parents: personality, attitudes, preferences, behavior, and cultural differences. Developmental Psychology, 31(5), 759-772.

Tyler, T. R. (1990). Why people obey the law. New Haven: Yale University Press.

UNICEF. (2001). A league table of child deaths by injury in rich nations. Innocenti report card $n^{\circ} 2$. Florence: UNICEF Innocenti Research Centre.

van Honk, J., Schutter, D. J. L. G., Hermans, E. J., Putman, P., Tuiten, A., \& Koppeschaar, H. (2004). Testosterone shifts the balance between sensitivity for punishment and reward in healthy young women. Psychoneuroendocrinology, 29, 937-943.

Waylen, A., \& McKenna, F. (2002). Cradle attitudes - grave consequences. The development of gender differences in risky attitudes and behaviour in road use. Reading University: Foundation for Road Safety Research.

Wood, W., \& Eagly, A. H. (2009). Gender identity. In M. Leary \& R. Hoyle (Eds.), Handbook of individual differences in social behavior (pp. 109-128). New York: Guilford.

Wood, W., \& Eagly, A. H. (2010). Gender. In S. T. Fiske, D. T. Gilbert \& G. Lindzey (Eds.), Handbook of social psychology (5th ed., vol. 1, pp. 629-667). New York: Wiley.

World Health Organization. (2002). Gender and Road Traffic Injuries. Geneva : Department of Gender and Women's Health.

Yagil, D. (1998). Gender and age-related differences in attitudes toward traffic laws and traffic violations. Transportation Research Part F : Traffic Psychology and Behaviour, 1, 123-135.

Yagil, D. (2000). Beliefs, motives and situational factors related to pedestrians' self-reported behavior at signal-controlled crossings. Transportation Research Part F: Traffic Psychology and Behaviour, 3(1), 1-13.

Yeung, N., \& von Hippel, C. (2008). Stereotype threat increases the likelihood that female drivers in a simulator run over jaywalkers. Accident Analysis \& Prevention, 40, 667-674.

Zahn-Waxler, C., \& Chapman, M. (1982). Immediate antecedents of caretakers' methods of discipline. Child Psychiatry and Human Development, 12, 179-192.

Zeedyk, M. S., \& Kelly, L. (2003). Behavioural observations of adult-child pairs at pedestrian crossings. Accident Analysis \& Prevention, 35(5), 771-776.

Zuckerman, M. (1991). Psychobiology of personality. Cambridge, England: Cambridge University Press. 
Zuckerman, M., Ball, S. A., \& Black, J. (1990). Influences of sensation seeking, gender, risk appraisal and situational motivation on smoking. Addictive Behaviors, 15, 209-220.

\section{NOTES}

1. http://lemanifeste.securite-routiere.gouv.fr/

\section{RÉSUMÉS}

Les données pointent une grande différence entre sexes en termes d'accidents dans l'espace routier chez les individus de sexe masculin, quel que soit leur âge, résultat d'une plus grande implication dans une large variété d'activités risquées et physiquement dangereuses. Cet article fait un état des lieux des connaissances permettant de comprendre le rapport entre genre et accidentologie routière dans une approche psycho-socio-développementale, en pointant les effets de la conformité aux stéréotypes de sexe sur le rapport au risque et sur la conformité aux règles, notamment en termes d'internalisation. Il propose des pistes de changement pour le continuum éducatif en sécurité routière afin d'augmenter l'internalisation des règles tout en prenant en compte les stéréotypes de sexe.

Accident data shows males, whatever their age, are significantly more involved in road crashes as e result of a larger involvement in a wide range of risky and physically dangerous activities. This paper takes stock of knowledge that enables to understand the effect of gender on road crashes in a psychosocial developmental approach, pointing out the effects of gender stereotype compliance on relation to risk and compliance with rules, particularly in terms of internalization. It proposes trail of changes for road safety education to better develop rule internalization while taking into account gender stereotypes.

\section{INDEX}

Mots-clés : genre, risque, conformité, stéréotypes, internalisation

Keywords : gender, risk, rules, compliance, stereotypes, internalization

\section{AUTEUR}

\section{MARIE-AXELLE GRANIE}

IFSTTAR-LMA (Institut Français des Sciences et Technologies des Transports, de l'Aménagement et des Réseaux, Laboratoire Mécanismes des Accidents) 